\title{
Remarks on the Pluricomplex Green Function
}

\author{
Marek JARnicki \& Peter Pflug
}

\begin{abstract}
We prove that the pluricomplex Green function $g$ and the Azukawa pseudometric $A$ have the product property on $G_{1} \times G_{2}$ if $G_{2}$ is pseudoconvex. Moreover, we show that $g_{G}$ and $A_{G}$ are upper semicontinuous for any domain $G \subset \mathbb{C}^{n}$. Explicit examples are given.
\end{abstract}

Let $G$ be a domain in $\mathbb{C}^{n}$. We put

$$
g_{G}(a, z):=\sup \left\{u(z): u \in \mathcal{K}_{G}(a)\right\}, \quad a, z \in G,
$$

where $\mathcal{K}_{G}(a)$ denotes the set of all logarithmically plurisubharmonic functions $u: G \rightarrow[0,1)$ such that there exist $M, r>0$, with $u(z) \leq M\|z-a\|$ for all $z \in B(a, r) \subset G\left(\|\|\right.$ denotes the Euclidean norm in $\mathbb{C}^{n}$ and $B(a, r):=\left\{z \in \mathbb{C}^{n}\right.$ : $\|z-a\|<r\})$. The function $g_{G}$ is called the pluricomplex Green function for $G$. It is known that $g_{G}(a, \cdot) \in \mathcal{K}_{G}(a)$ (cf. [Jar-Pfl], Lemma 4.2.3). Let $A_{G}$ denote the Azukawa pseudometric for $G$ given by the formula

$$
A_{G}(a, X):=\limsup _{\lambda \rightarrow 0} \frac{1}{|\lambda|} g_{G}(a, a+\lambda X), \quad a \in G, X \in \mathbb{C}^{n} .
$$

Define

$$
\begin{array}{r}
\tilde{k}_{G}^{*}(a, z):=\inf \{\sigma \in[0,1): \exists \varphi \in \mathcal{O}(E, G): \varphi(0)=a, \varphi(\sigma)=z\}, \quad a, z \in G, \\
\kappa_{G}(a ; X):=\inf \left\{\sigma \in[0,+\infty): \exists \varphi \in \mathcal{O}(E, G): \varphi(0)=a, \sigma \cdot \varphi^{\prime}(0)=X\right\}, \\
a \in G, X \in \mathbb{C}^{n},
\end{array}
$$

where $E$ denotes the unit disc in $\mathbb{C}$. The function $\tanh ^{-1}\left(\tilde{k}_{G}^{*}\right)$ is called the Lempert function; $\kappa_{G}$ is the Kobayashi-Royden pseudometric.

Recall that the systems $\left(g_{G}\right)_{G}$ and $\left(\tilde{k}_{G}^{*}\right)_{G}$ are $m$-contractible families of functions; the systems $\left(A_{G}\right)_{G}$ and $\left(\kappa_{G}\right)_{G}$ are contractible families of pseudometrics (cf. [Jar-Pfl]). 
1. The upper semicontinuity. In all papers discussing the fundamental properties of $g_{G}$ and $A_{G}$ the following theorem can be found (cf. [Kli 1-3], [Jar-Pfl]).

If $G$ is pseudoconvex, then $g_{G}$ and $A_{G}$ are upper semicontinuous.

The proof of this result is based on the continuity of $g_{G}$ for hyperconvex domains.

One might get the impression that the assumption ' $G$ is pseudoconvex' is somehow important. This is not so. We will show by elementary methods that $g_{G}$ and $A_{G}$ are upper semicontinuous for arbitrary domains $G$.

Theorem 1. For an arbitrary domain $G \subset \mathbb{C}^{n}$ the pluricomplex Green function $g_{G}: G \times G \rightarrow[0,1)$ and the Azukawa pseudometric $A_{G}: G \times \mathbb{C}^{n} \rightarrow$ $[0,+\infty)$ are upper semicontinuous.

Proof. First, let us recall some known facts (cf. [Jar-Pfl], Sections 4.1, 4.2; see also [Kli 2]).

(a) If $g_{G}$ is upper semicontinuous, then $A_{G}$ is upper semicontinuous.

(b) If $G$ is bounded, then for any ball $B(a, r) \subset G$ and for any $\varepsilon>0$ there exists $\delta \in(0, r)$ such that

$$
\left[g_{G}(x, z)\right]^{1+\varepsilon} \leq g_{G}(a, z), \quad x \in B(a, \delta), z \in G \backslash B(a, r) .
$$

(c) If $G_{\nu} \nearrow G$, then $g_{G_{\nu}} \searrow g_{G}$.

(d) $g_{G}$ is continuous at all points $(a, a) \in G \times G$.

By virtue of (a) and (c), we can restrict ourselves to the case where $G$ is bounded. Because of $(\mathrm{d})$, it suffices to prove that $g_{G}$ is upper semicontinuous at any point $(a, b) \in G \times G$ with $a \neq b$. Fix $r>0$ such that $b \notin \bar{B}(a, r) \subset G$. Suppose that $g_{G}(a, b)<\alpha<\beta$ and let $\varepsilon>0$ be such that $\alpha<\beta^{1+\varepsilon}$. Take $\delta \in(0, r)$ as in (b). Since the function $g_{G}$ is upper semicontinuous (recall that $\left.g_{G}(a, \cdot) \in \mathcal{K}_{G}(a)\right)$, there exists $\eta>0$ such that $g_{G}(a, z)<\alpha, z \in B(b, \eta) \subset$ $G \backslash B(a, r)$. Finally,

$$
g_{G}(x, z) \leq\left[g_{G}(a, z)\right]^{1 /(1+\varepsilon)}<\alpha^{1 /(1+\varepsilon)}<\beta, \quad x \in B(a, \delta), z \in B(b, \eta),
$$

which completes the proof.

\section{Remarks.}

(a) Observe that the same argument shows that if $G$ is bounded and if $g_{G}(a, \cdot)$ is continuous at $b$, then $g_{G}$ is continuous at $(a, b)$. The implication is not true if $G$ is unbounded (cf. [Jar-Pfl], Proposition 4.4.2).

(b) Using the description of $g_{G}$ proposed by Poletsky in [Pol], one can give another proof of Theorem 1.

(c) The fact that $A_{G}$ is upper semicontinuous permits us to define the integrated form of $A_{G}$ and next to apply the general theory to study this object (see [Jar-Pfl], Section 4.3, for more details). 
2. The product property. Let $G_{j} \subset \mathbb{C}^{n_{j}}, j=1,2$, be arbitrary domains. We say that $g$, respectively $A$, has the product property on $G_{1} \times G_{2}$ if

$$
g_{G_{1} \times G_{2}}=\max \left\{g_{G_{1}}, g_{G_{2}}\right\}
$$

respectively,

$$
A_{G_{1} \times G_{2}}=\max \left\{A_{G_{1}}, A_{G_{2}}\right\} .
$$

It is known that (2) is a consequence of (1) (cf. [Jar-Pfl], Remark 9.4(a)). Moreover, the product properties hold if $G_{j}, j=1,2$, are pseudoconvex; the proof is based on the boundary behavior of $g_{G}$ for hyperconvex domains, the MongeAmpère equation, and the domination principle (cf. [Jar-Pfl], Theorem 9.8). We would like to point out that in general there is no simple relation between $g_{G}$ and $g_{\tilde{G}}$, where $\tilde{G}$ is the envelope of holomorphy of $G$ (cp. Section 3 ).

In this note we will prove (Theorem $2(\mathrm{a})$ ) that the product property is also true on $G_{1} \times G_{2}$ if $G_{2}$ is pseudoconvex (without assuming $G_{1}$ to be pseudoconvex). The more precise formulation of our result is as follows.

Theorem 2. Let $G_{j} \subset \mathbb{C}^{n_{j}}, j=1,2$, be domains.

(a) If $G_{2}$ is pseudoconvex, then $g$ and $A$ have the product properties on $G_{1} \times G_{2}$.

(b) Assume that for $b, w_{0} \in G_{2}$ we have

$$
g_{G_{2}}\left(b, w_{0}\right)=\tilde{k}_{G_{2}}^{*}\left(b, w_{0}\right) .
$$

Then,

$$
g_{G_{1} \times G_{2}}\left((a, b),\left(z, w_{0}\right)\right)=\max \left\{g_{G_{1}}(a, z), g_{G_{2}}\left(b, w_{0}\right)\right\}, \quad a, z \in G_{1} .
$$

(c) Assume that for $b \in G_{2}, Y_{0} \in \mathbb{C}^{n_{2}}$ we have

$$
A_{G_{2}}\left(b ; Y_{0}\right)=\kappa_{G_{2}}\left(b ; Y_{0}\right) .
$$

Then,

$$
A_{G_{1} \times G_{2}}\left((a, b) ;\left(X, Y_{0}\right)\right)=\max \left\{A_{G_{1}}(a ; X), A_{G_{2}}\left(b ; Y_{0}\right)\right\}, \quad a \in G_{1}, X \in \mathbb{C}^{n_{1}} .
$$

To prove Theorem 2, it is sufficient to verify the following lemma.

Lemma 3. The product properties hold on $G \times E$ for arbitrary domain $G \subset \mathbb{C}^{n}$. 
Proof that Lemma $3 \Rightarrow$ Theorem 2. For (a): We already know that it suffices to prove the product property for $g$.

Let $\left(G_{2, \nu}\right)_{\nu \in \mathbb{N}}$ be a sequence of bounded hyperconvex domains such that $G_{2, \nu} \nearrow G_{2}$. Then $g_{G_{2, \nu}} \searrow g_{G_{2}}$ and $g_{G_{1} \times G_{2, \nu}} \searrow g_{G_{1} \times G_{2}}$, and therefore, we assume that $G_{2}$ is bounded hyperconvex. By virtue of Lemma 3 we only have to show that

$$
\begin{aligned}
& g_{G_{1} \times G_{2}}((a, b),(z, w)) \leq g_{G_{1} \times E}\left((a, 0),\left(z, g_{G_{2}}(b, w)\right)\right) \\
& \quad \text { for all }(a, b),(z, w) \in G_{1} \times G_{2} .
\end{aligned}
$$

Fix $(a, b) \in G_{1} \times G_{2}$. Recall (Lemma 3$)$ that

$$
g_{G_{1} \times E}((a, 0),(z, \lambda))=\max \left\{g_{G_{1}}(a, z),|\lambda|\right\}, \quad(z, \lambda) \in G_{1} \times E .
$$

In particular, for each $z \in G_{1}$, the function $g_{G_{1} \times E}((a, 0),(z, \cdot))$ is continuous on $E$. Consequently, inequality (3) follows from:

$$
\begin{aligned}
& g_{G_{1} \times G_{2}}((a, b),(z, w)) \leq g_{G_{1} \times E}((a, 0),(z, \lambda)) \\
& \quad \text { for all }(z, w, \lambda) \in G_{1} \times G_{2} \times E \text { with } g_{G_{2}}(b, w)<|\lambda| .
\end{aligned}
$$

Observe that for fixed $(w, \lambda) \in G_{1} \times E$ both sides of (4) are plurisubharmonic on $G_{1}$. Thus, it is enough to show that there exists a subset $S \subset G_{1}$ of zero Lebesgue measure such that

$$
\begin{aligned}
& g_{G_{1} \times G_{2}}((a, b),(z, w)) \leq g_{G_{1} \times E}((a, 0),(z, \lambda)) \\
& \quad \text { for all }(z, w, \lambda) \in\left(G_{1} \backslash S\right) \times G_{2} \times E \text { with } g_{G_{2}}(b, w)<|\lambda| .
\end{aligned}
$$

Let

$$
\begin{array}{rlrl}
\varphi(w) & :=\log g_{G_{2}}(b, w), & w \in G_{2}, \\
V(z, w) & :=\log g_{G_{1} \times G_{2}}((a, b),(z, w)), & (z, w) \in G_{1} \times G_{2}, \\
u(z, \zeta) & :=\sup \left\{V(z, w):(z, w) \in G_{1} \times G_{2}, \varphi(w)<\operatorname{Re} \zeta\right\}, \\
u_{0}(z, \lambda) & :=\exp (u(z, \log |\lambda|)), & (z, \zeta) \in G_{1} \times H, \\
& (z, \lambda) \in G_{1} \times E_{*},
\end{array}
$$

where $H:=\{\zeta \in \mathbb{C}: \operatorname{Re} \zeta<0\}$ and $E_{*}:=E \backslash\{0\}$.

Note that: 
- $\varphi$ is a continuous plurisubharmonic function with $\left\{w \in G_{2}: \varphi(w)<t\right\} \Subset G_{2}$ for negative $t$, and $\left(d d^{c}\right)^{n_{2}} \varphi \equiv 0$ in $G_{2} \backslash\{0\}$,

- $V$ is plurisubharmonic,

- $u(z, \zeta)=u(z, \operatorname{Re} \zeta),(z, \zeta) \in G_{1} \times H$.

To get (5) it suffices to prove that

$$
u_{0}(z, \lambda) \leq g_{G_{1} \times E}((a, 0),(z, \lambda)), \quad(z, \lambda) \in\left(G_{1} \backslash S\right) \times E_{*} .
$$

By [Dem], Corollary 6.6(b), for each $z \in G_{1}$, the function $u(z, \cdot)$ is continuous on $H$. Hence, inequality (6) is equivalent to:

$$
u_{0}(z, \lambda) \leq g_{G_{1} \times E}((a, 0),(z, \lambda)) \quad \text { for a.a. }(z, \lambda) \in G_{1} \times E_{*} .
$$

Let $M>0, r \in(0,1)$ be such that

$$
\begin{aligned}
& g_{G_{1} \times G_{2}}((a, b),(z, w)) \leq M(\|z-a\|+\|w-b\|) \\
& \qquad \text { for all }(z, w) \in B(a, r) \times B(b, r) \subset G_{1} \times G_{2} .
\end{aligned}
$$

Choose $R \geq 1$ such that $G_{2} \subset B(b, R)$. Then,

$$
g_{G_{2}}(b, w) \geq \frac{\|w-b\|}{R}, \quad w \in G_{2},
$$

and, consequently,

$$
u_{0}(z, \lambda) \leq M R(\|z-a\|+|\lambda|), \quad z \in B(a, r), 0<|\lambda|<\frac{r}{R} .
$$

We claim that the function $u$ is upper semicontinuous on $G_{1} \times H$. Let $G_{1} \times H \ni\left(z_{\nu}, \zeta_{\nu}\right) \rightarrow\left(z_{0}, \zeta_{0}\right) \in G_{1} \times H$ and let $w_{\nu} \in G_{2}$ be such that $\varphi\left(w_{\nu}\right) \leq$ $\operatorname{Re} \zeta_{\nu}, u\left(z_{\nu}, \zeta_{\nu}\right)=V\left(z_{\nu}, w_{\nu}\right), \nu \in \mathbb{N}$. We may assume that $w_{\nu} \rightarrow w_{0} \in G_{2}$ with $\varphi\left(w_{0}\right) \leq \operatorname{Re} \zeta_{0}$. Hence,

$$
\limsup _{\nu \rightarrow \infty} u\left(z_{\nu}, \zeta_{\nu}\right)=\limsup _{\nu \rightarrow \infty} V\left(z_{\nu}, w_{\nu}\right) \leq V\left(z_{0}, w_{0}\right) \leq u\left(z_{0}, \zeta\right)
$$

$$
\text { whenever } \operatorname{Re} \zeta_{0}<\operatorname{Re} \zeta<0 .
$$

Then continuity of $u\left(z_{0}, \cdot\right)$ gives the required result.

By Theorem 6.11 in [Dem] the function $u$ is weakly plurisubharmonic on $G_{1} \times H$, i.e., there exists a plurisubharmonic function $\tilde{u}$ on $G_{1} \times H$ such that $\tilde{u}=u$ a.e. on $G_{1} \times H$. Since $u$ is upper semicontinuous, $\tilde{u} \leq u$ on $G_{1} \times H$.

Observe that $\tilde{u}(z, \zeta)=\tilde{u}(z, \operatorname{Re} \zeta),(z, \zeta) \in G_{1} \times H$. For fix $\zeta_{0} \in H$. Then the two plurisubharmonic functions

$$
(z, \zeta) \rightarrow \tilde{u}\left(z, \zeta_{0}+\zeta\right) \quad \text { and } \quad(z, \zeta) \rightarrow \tilde{u}\left(z, \operatorname{Re} \zeta_{0}+\zeta\right)
$$


coincide a.a. on $G_{1} \times\left\{\zeta \in \mathbb{C}: \operatorname{Re}\left(\zeta+\zeta_{0}\right)<0\right\}$. Therefore, they coincide everywhere.

Define $\tilde{u}_{1}(z, \lambda):=\tilde{u}(z, \log \lambda),(z, \lambda) \in G_{1} \times E_{*}$. It is clear that $\tilde{u}_{1}$ is welldefined, plurisubharmonic, and $\tilde{u}_{1}<0$. We extend $\tilde{u}_{1}$ plurisubharmonically on the whole $G_{1} \times E$ setting,

$$
\tilde{u}_{1}(z, 0):=\limsup _{G_{1} \times E_{*} \ni\left(z^{\prime}, \lambda\right) \rightarrow(z, 0)} \tilde{u}_{1}\left(z^{\prime}, \lambda\right), \quad z \in G_{1} .
$$

Define $\tilde{u}_{0}:=\exp \left(\tilde{u}_{1}\right)$. Then $\tilde{u}_{0}: G_{1} \times E \rightarrow[0,1)$ is a log-plurisubharmonic function and $\tilde{u}_{0} \leq u_{0}$ on $G_{1} \times E_{*}$. In particular, by (8) we have

$$
\tilde{u}_{0}(z, \lambda) \leq M R(\|z-a\|+|\lambda|), \quad z \in B(a, r), 0<|\lambda|<\frac{r}{R} .
$$

Since both sides are plurisubharmonic on $G_{1} \times E$, the inequality above holds for all $z \in B(a, r)$ and $|\lambda|<r / R$.

Thus $\tilde{u}_{0} \in \mathcal{K}_{G_{1} \times E}((a, 0))$ and therefore, $\tilde{u}_{0} \leq g_{G_{1} \times E}((a, 0), \cdot)$. Since $\tilde{u}_{0}=$ $u_{0}$ a.e. on $G_{1} \times E_{*}$, we end up with (7), so the proof of (a) is complete.

For part (b): Fix $a, z \in G_{1}$. Choose a sequence of holomorphic mappings $\varphi_{\nu}: E \rightarrow G_{2}$ with $\varphi_{\nu}(0)=b$ and $\varphi_{\nu}\left(\sigma_{\nu}\right)=w_{0}$ for some $\sigma_{\nu} \in[0,1)$ such that $\sigma_{\nu} \searrow \tilde{k}_{G_{2}}^{*}\left(b, w_{0}\right)=g_{G_{2}}\left(b, w_{0}\right)$. Using contractibility and Lemma 3 gives

$$
\begin{aligned}
g_{G_{1} \times G_{2}}\left((a, b),\left(z, w_{0}\right)\right) & \leq g_{G_{1} \times E}\left((a, 0),\left(z, \sigma_{\nu}\right)\right) \\
& =\max \left\{g_{G_{1}}(a, z), \sigma_{\nu}\right\} \searrow \max \left\{g_{G_{1}}(a, z), g_{G_{2}}\left(b, w_{0}\right)\right\} .
\end{aligned}
$$

Recall that the remaining inequality is always satisfied.

The proof of (c) is analogous.

Lemma 3 is a consequence of the following lemma.

Lemma 4. Let $G \subset \mathbb{C}^{n}$ be a bounded domain. Then, for $a, z \in G, \lambda \in E$ such that $g_{G}(a, z)=|\lambda|$ we have:

$$
g_{G \times E}((a, 0),(z, \lambda)) \leq|\lambda| .
$$

Proof that Lemma $4 \Rightarrow$ Lemma 3. It suffices to consider only the case where $G$ is bounded and to show that for arbitrary $a, z_{0} \in G$ with $a \neq z_{0}$ we have

$$
g_{G \times E}\left((a, 0),\left(z_{0}, \lambda\right)\right) \leq \max \left\{g_{G}\left(a, z_{0}\right),|\lambda|\right\}, \quad \lambda \in E .
$$

If $|\lambda| \leq g_{G}\left(a, z_{0}\right)$, then (10) follows from (9) and the maximum principle for subharmonic functions. If $g_{G}\left(a, z_{0}\right)<|\lambda|<1$, then (10) is a consequence of (9) and the maximum principle applied to the subharmonic function

$$
\lambda \rightarrow \log g_{G \times E}\left((a, 0),\left(z_{0}, \lambda\right)\right)-\log |\lambda| .
$$

Finally, Lemma 4 follows directly from the fact (Lemma 5 ) that the function

$$
G \ni z \rightarrow g_{G \times E}\left((a, 0),\left(z, g_{G}(a, z)\right)\right) \in[0,1)
$$

belongs to $\mathcal{K}_{G}(a)$. 
Lemma 5. Let $G \subset \mathbb{C}^{n}$ be a domain and let $a \in G$. Then the function $u$ given by the formula

$$
u(z):=g_{G \times E}\left((a, 0),\left(z, g_{G}(a, z)\right)\right), \quad z \in G,
$$

is of the class $\mathcal{K}_{G}(a)$.

\section{Proof.}

STEP 1. For any point $z \in G$ the subharmonic function $g_{G \times E}((a, 0),(z, \cdot))$ is invariant under rotations, i.e.,

$$
g_{G \times E}((a, 0),(z, \lambda))=g_{G \times E}((a, 0),(z,|\lambda|)), \quad \lambda \in E .
$$

Hence, the function

$$
[0,1) \ni t \rightarrow g_{G \times E}((a, 0),(z, t))
$$

is increasing and continuous (cf. [Kli 2]).

STEP 2. The monotonicity from Step 1 (together with the fact that the functions $g_{G \times E}((a, 0),(\cdot, \cdot))$ and $g_{G}(a, \cdot)$ are upper semicontinuous) implies that $u$ is upper semicontinuous on $G$. Moreover, since $g_{G \times E}((a, 0),(\cdot, \cdot)) \in \mathcal{K}_{G \times E}((a, 0))$ and $g_{G}(a, \cdot) \in \mathcal{K}_{G}(a)$, we see that there exist $M, r>0$ with $u(z) \leq M\|z-a\|$ for all $z \in B(a, r) \subset G$.

It remains to prove that $u$ is logarithmically plurisubharmonic.

SteP 3. Fix $z_{0} \in G$ and $B\left(z_{0}, r\right) \Subset G$. Choose a sequence $\left(h_{\nu}\right)_{\nu=1}^{\infty}$ of negative continuous plurisubharmonic functions on $B\left(z_{0}, r\right)$ such that $h_{\nu} \searrow$ $\log g_{G}(a, \cdot)$ on $B(a, r)$. Then, $\tilde{h}_{\nu}:=\exp \left(h_{\nu}\right) \searrow g_{G}(a, \cdot)$. Put

$$
v_{\nu}(z):=\log g_{G \times E}\left((a, 0),\left(z, \tilde{h}_{\nu}(z)\right)\right), \quad z \in B\left(z_{0}, r\right) .
$$

In view of Step 1 , we get that $v_{\nu} \searrow \log u$ on $B\left(z_{0}, r\right)$.

Thus, it remains to show that all the functions $v_{\nu}$ are plurisubharmonic near $z_{0}$.

Fix $\nu$. Applying the theorem of Bremermann (cf. [Sib]), we find sequences $\left(f_{j}\right)_{j=1}^{\infty} \subset \mathcal{O}\left(B\left(z_{0}, r / 2\right)\right),\left(c_{j}\right)_{j=1}^{\infty} \subset(0,+\infty)\left(f_{j} \not \equiv 0, j \in \mathbb{N}\right)$ such that

$$
h_{\nu}=\sup _{j \in \mathbb{N}}\left\{c_{j} \log \left|f_{j}\right|\right\} \quad \text { i.e., } \quad \tilde{h}_{\nu}=\sup _{j \in \mathbb{N}}\left\{\left|f_{j}\right|^{c_{j}}\right\}<1 \quad \text { on } B\left(z_{0}, \frac{r}{2}\right) .
$$


Hence, by virtue of Step 1, we have

$$
v_{\nu}(z)=\sup _{j \in \mathbb{N}}\left\{\log g_{G \times E}\left((a, 0),\left(z,\left|f_{j}(z)\right|^{c_{j}}\right)\right)\right\}, \quad z \in B\left(z_{0}, \frac{r}{2}\right) .
$$

Since $v_{\nu}$ is an upper semicontinuous function, it suffices to show that all the upper semicontinuous functions

$$
B\left(z_{0}, \frac{r}{2}\right) \ni z \stackrel{\psi_{j}}{\rightarrow} \log g_{G \times E}\left((a, 0),\left(z,\left|f_{j}(z)\right|^{c_{j}}\right)\right)
$$

are plurisubharmonic. In view of Step 1 , we see that $\psi_{j}$ is plurisubharmonic on $B\left(z_{0}, r / 2\right) \backslash Z_{j}$, where $Z_{j}:=\left\{z \in B\left(z_{0}, r / 2\right): f_{j}(z)=0\right\}$. Since the set $Z_{j}$ is analytic, $\psi_{j}$ can be continued as a plurisubharmonic function $\tilde{\psi}_{j}$ on the whole $B\left(z_{0}, r / 2\right)$. We only need to prove that $\tilde{\psi}_{j}=\psi_{j}$ on $Z_{j}$. Take a point $z^{*} \in Z_{j}$. Using the plurisubharmonicity of the function $z \rightarrow \log g_{G \times E}((a, 0),(z, 0))$, it follows (again by Step 1) that

$$
\begin{aligned}
\psi_{j}\left(z^{*}\right) & =\limsup _{Z_{j} \not z \rightarrow z^{*}} \log g_{G \times E}((a, 0),(z, 0)) \\
& \leq \limsup _{Z_{j} \not z \rightarrow z^{*}} \log g_{G \times E}\left((a, 0),\left(z,\left|f_{j}(z)\right|^{c_{j}}\right)\right) \\
& =\limsup _{Z_{j} \not z \rightarrow z^{*}} \psi_{j}(z) \\
& =\tilde{\psi}_{j}\left(z^{*}\right) \leq \psi_{j}\left(z^{*}\right) .
\end{aligned}
$$

\section{Remark.}

(a) We would like to point out that the proof of Theorem 2(a) given here is independent of Theorem 9.8 in [Jar-Pfl].

(b) A. Edigarian informed us that, using the description of $g_{G}$ from [Pol], one can give another proof of Theorem $2(\mathrm{~b})$.

Remark. Having Theorem 2, we think that the product property for $g$ (and $A$ ) is true for arbitrary domains.

3. An example. In [Jar-Pfl], Example 4.2.8, and in [Kli 3], explicit formulas for $g_{G}$ for certain Reinhardt domains are given. The following more general example contains, in particular, these results.

Example. Let $0 \leq a_{1}<a_{2} \leq 1,0 \leq b_{1}<b_{2} \leq 1, Q:=\left[a_{1}, a_{2}\right] \times\left[b_{1}, b_{2}\right]$ be such that $(0,0) \notin Q$. Put

$$
G:=\left\{\left(z_{1}, z_{2}\right) \in E \times E:\left(\left|z_{1}\right|,\left|z_{2}\right|\right) \notin Q\right\} .
$$

There are two cases which surprisingly give two different types of Green functions. 
CASE 1. $\stackrel{\circ}{Q} \cap\{(t, t): t \in[0,1]\}=\varnothing$. Then,

$$
g_{G}\left((0,0),\left(z_{1}, z_{2}\right)\right)=\max \left\{\left|z_{1}\right|,\left|z_{2}\right|\right\} .
$$

CASE 2. $\stackrel{\circ}{Q} \cap\{(t, t): t \in[0,1]\} \neq \varnothing$. Then,

$$
g_{G}\left((0,0),\left(z_{1}, z_{2}\right)\right)
$$$$
=\max \left\{\left|z_{1}\right|,\left|z_{2}\right|,\left(e^{\log \left(b_{2} / a_{1}\right) \log \left(a_{2} / b_{1}\right)}\left|z_{1}\right|^{\log \left(a_{2} / b_{1}\right)}\left|z_{2}\right|^{\log \left(b_{2} / a_{1}\right)}\right)^{1 / \log \left(a_{2} b_{2} / a_{1} b_{1}\right)}\right\}
$$$$
\text { if }\left|z_{1}\right|<a_{1} \text { or }\left|z_{2}\right|<b_{1} \text {; }
$$

$$
\begin{aligned}
& g_{G}\left((0,0),\left(z_{1}, z_{2}\right)\right) \\
= & \max \left\{\left|z_{1}\right|,\left|z_{2}\right|,\left(\left|z_{1}\right|^{\log b_{2} \log \left(b_{1} / a_{2}\right)}\left|z_{2}\right|^{\log a_{2} \log \left(a_{1} / b_{2}\right)}\right)^{1 /\left(\log a_{1} \log b_{1}-\log a_{2} \log b_{2}\right)}\right\} \\
& \text { if }\left|z_{1}\right|>a_{2} \text { or }\left|z_{2}\right|>b_{2} .
\end{aligned}
$$

For $a_{1}=0$ (resp. $\left.b_{1}=0\right)$, the formulas should be understood as limits.

The proof of the formulas above is based on the Schwarz Lemma and the Hadamard Three Circles Theorem for subharmonic functions.

\section{REFERENCES}

[Dem] J.-P. Demailly, Measures de Monge-Ampere et caractérisation géométrique des variétés algébriques affines, Mém. Soc. Math. France (N. S.) 19 (1985), 1-125.

[Jar-Pfl] M. JARniCKI \& P. Pflug, Invariant Distances and Metrics in Complex Analysis, de Gruyter Expositions in Mathematics 9, Walter de Gruyter, 1993.

[Kli 1] M. KLIMEK, Infinitesimal pseudometrics and the Schwarz Lemma, Proc. Amer. Math. Soc. 105 (1989), 134-140.

[Kli 2] — Pluripotential Theory, Oxford University Press, 1991.

[Kli 3] , Invariant pluricomplex Green functions, Banach Center Publications (to appear).

[Pol] E. A. Poletsky, Holomorphic currents, Indiana Univ. Math. J. 42 (1993), 85-144.

[Sib] N. SibOny, Prolongement de fonctions holomorphes bornées et metrique de Carathéodory, Invent. Math. 29 (1975), 205-230.

\section{MAREK JARNICKI}

Uniwersytet Jagiellonski

Instytut Matematyki

Reymonta 4

30-059 Kraków, Poland

E-MAIL: jarnicki@im.uj.edu.pl

\section{Peter Pflug}

Carl von Ossietzky Universität Oldenburg Fachbereich Mathematik

Postfach 2503

D-26111 Oldenburg, Germany

E-MAIL: pflug@hrz2.pcnet.uni-oldenburg.de

Received: November 3rd, 1994; revised: April 10th, 1995. 